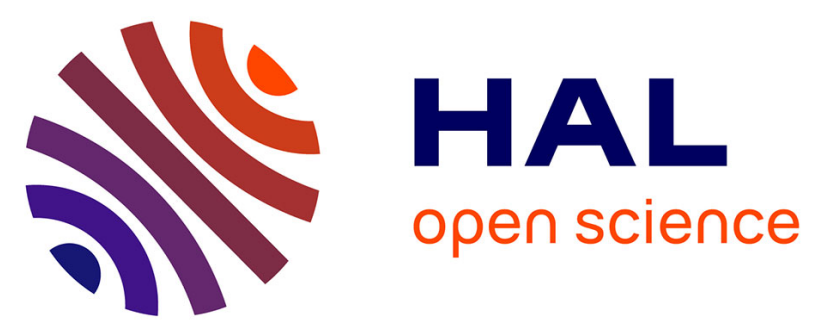

\title{
Variation géographique des nombres chromosomiques de base et polyploïdie dans le genre Dioscorea à propos du dénombrement des espèces transversa Brown, pilosiuscula Bert. et trifida $L$
}

Sadi Essad, Claire Maunoury

\section{- To cite this version:}

Sadi Essad, Claire Maunoury. Variation géographique des nombres chromosomiques de base et polyploïdie dans le genre Dioscorea à propos du dénombrement des espèces transversa Brown, pilosiuscula Bert. et trifida L. Agronomie, 1984, 4 (7), pp.611-617. hal-00884679

\section{HAL Id: hal-00884679 \\ https://hal.science/hal-00884679}

Submitted on 1 Jan 1984

HAL is a multi-disciplinary open access archive for the deposit and dissemination of scientific research documents, whether they are published or not. The documents may come from teaching and research institutions in France or abroad, or from public or private research centers.
L'archive ouverte pluridisciplinaire HAL, est destinée au dépôt et à la diffusion de documents scientifiques de niveau recherche, publiés ou non, émanant des établissements d'enseignement et de recherche français ou étrangers, des laboratoires publics ou privés. 


\title{
Variation géographique des nombres chromosomiques de base et polyploïdie dans le genre Dioscorea à propos du dénombrement des espèces transversa Brown, pilo- siuscula Bert. et trifida L.
}

\author{
Sadi ESSAD \\ avec la collaboration technique de Claire MAUNOURY \\ I.N.R.A., Station de Génétique et d'Amélioration des Plantes, Route de St-Cyr, F 78000 Versailles
}

RÉSUMÉ

Les dénombrements chromosomiques de l'espèce océanienne Dioscorea transversa Brown cv. « Waël " ainsi que de 11 cultivars d'origines diverses de $D$. trifida $\mathrm{L}$. mettent en évidence un nombre $2 \mathrm{n}=80$. D. pilosiuscula Bert. présente des difficultés particulières de dénombrement chromosomique qui ne nous permettent qu'une estimation du nombre $2 \mathrm{n}$ qui est d'environ 80 chromosomes.

Un tableau récapitulatif, que nous avons souhaité exhaustif, des dénombrements publiés pour le genre Dioscorea (72 espèces dénombrées sur un total estimé à 620 , soit environ $12 \mathrm{p}$. 100 de l'ensemble des espèces du genre) permet de préciser les modalités de son évolution chromosomique. A partir de 2 nombres de base $(x=9$ et 10$)$, la polyploïdie est intervenue dans ce genre d'une façon particulièrement extensive. Les formes $4 \mathrm{x}$ apparaissent comme les plus nombreuses, puis à égalité, les formes $2 \mathrm{x}, 6 \mathrm{x}$ et $8 \mathrm{x}$. Tous les niveaux intermédiaires se rencontrent jusqu'à au moins $10 x$. La variation polyploïde rencontrée à l'intérieur même de certaines espèces et l'absence apparente de relation entre polyplö̈die et valeur alimentaire des espèces conduisent cependant à douter d'une réelle intervention de la variation polyploïde sur la spéciation du genre.

Le nombre chromosomique de base $\mathrm{x}=10$ qui caractérise toutes les espèces asiatiques ne concerne que 52 p. 100 des espèces africaines et 13 p. 100 de l'ensemble des espèces américaines, les espèces complémentaires présentant $\mathrm{x}=9$. Il faut remarquer que les 80 chromosomes de $D$. trifida $\mathrm{L}$. établissent l'existence du nombre de base $\mathrm{x}=10$ parmi les espèces américaines pour lesquelles il demeurait encore contesté.

Geographical variation in base chromosome number and polyploidy within the genus Dioscorea, on the basis of chromosome counts in D. transversa Brown, D. pilosiuscula Bert. and D. trifida $L$.

Chromosome counts in Dioscorea trifida L. (11 cultivars) and D. transversa Brown give $2 \mathrm{n}=80 . D$. pilosiuscula Bert. have approximately the same number.

A summary table, as 'complete as possible, of the published chromosome numbers in Dioscorea $(72$ species out of a total of 620 estimated, i.e. about $12 \%$ of the whole genus) provides a basis for hypotheses on chromosome evolution in the genus. It would seem that polyploidy operated particularly actively in this genus, with 2 base numbers $(x=9$ and 10$) ; 4 x$ forms are the most numerous, followed by $2 x, 6 x$ and $8 x$ forms in the same proportions. All the intermediate levels appear up to $10 \mathrm{x}$ at least. However, the variation in polyploidy observed within some species and the lack of obvious relations between polyploidy and food value in yam species suggest that polyploid variation has not actually been involved in speciation in this genus. The base chromosome number $\mathrm{x}=10$ found, till now, in all the Asiatic species, concerns only 52 per cent of the African species and 13 per cent of the American species examined, the others having $x=9$. The demonstration that $D$. trifida L. has 80 chromosomes establishes the existence of the base number $\mathrm{x}=10$ in American species, which was previously in doubt.

\section{INTRODUCTION}

Classé dans les Monocotylédones, le genre Dioscorea $L$. présente cependant un certain nombre de caractères appartenant habituellement aux Dicotylé- dones. Ces caractères sont énumérés par DEGRAS et al. (1977). Nous y ajouterons la cytodiérèse simultanée des 4 cellules filles en fin de méiose mâle, au moins chez D. trifida L. Depuis la dernière monographie du genre (KNUTH, 1924 in COURSEY, 1967) on 
admet qu'il regroupe plus de 600 espèces. C'est dire la richesse botanique considérable du genre Dioscorea, essentiellement intertropical, qui s'étend sur les 5 continents et contribue à nourrir quelques dizaines de millions d'hommes. Déjà très répandu à la fin de l'ère secondaire avec un centre d'origine que l'on situe en Asie du Sud-Est, son évolution s'est trouvée profondément marquée par les grands événements géologiques qui ont conduit à la séparation actuelle des continents : séparation de l'Amérique du Sud à la fin du Crétacé puis, au Pliocène, isolement des 3 continents de l'Ancien Monde par la formation de la Méditerranée et des déserts du Moyen-Orient. Trois centres de dispersion principaux subsistent que l'on caractérise par les espèces et par les nombres chromosomiques. Ces centres de dispersion sont situés en Asie, où $x=10$, en Afrique où $x=9$ et 10 et en Amérique où $\mathrm{x}$ était jusqu'ici considéré comme étant uniquement de 9 chromosomes (MARTIN \& ORTIZ, 1963 ; COURSEY, 1976). D'autre part, l'isolement évolutif de chacun de ces centres de dispersion est tel que même les sections du genre ne réunissent que très exceptionnellement des espèces issues de continents différents : sur 23 sections se répartissant les espèces de l'Ancien Monde, 3 seulement regroupent des espèces asiatiques et africaines. Ces 3 sections sont d'ailleurs remarquables par le fait qu'elles renferment les espèces alimentaires les plus importantes économiquement. Ce sont $D$. alata L., opposita Thumb. et japonica Thumb. en Asie, cayenensis Lamk. ssp. cayenensis et rotundata Poir. en Afrique et nummularia Lamk. (Océanie) pour la section ENANTIOPHYLLUM; hispida Dennst. et pentaphylla L. en Asie et dumetorum Pax. en Afrique pour la section LASIOPHYTON : bulbifera $L$. (Asie et Afrique) pour la section OPSOPHYTON. La liste des espèces alimentaires essentielles supposerait que l'on ajoute seulement à la précédente, pour qu'elle soit complète, l'espèce asiatique esculenta (Lour.) Burk. (section COMBILIUM) et l'espèce américaine trifida $\mathrm{L}$. de la section MACROGYNODIUM. Remarquons que les espèces océaniennes sont considérées comme dérivant directement du groupe asiatique. Pour tous ces aspects, on pourra notamment consulter avec intérêt les ouvrages et publications de AYENSU \& COURSEY (1972) ; BURKILL (1960) ; COURSEY $(1967,1976)$.

La liste des nombres chromosomiques, qui s'est trouvée sensiblement enrichie depuis le tableau récapitulatif publié par MARTIN \& ORTIZ (1963), méritait d'être remise à jour et discutée. C'est ce que nous nous sommes efforcé de faire à l'occasion de certains dénombrements, nouveaux ( $D$. transversa Brown et pilosiuscula Bert.) ou en désaccord avec les données bibliographiques (D. trifida L.).

\section{MATÉRIEL ET MÉTHODES}

Les dénombrements réalisés dans le cours du présent travail, effectué à l'initiative de L. DEGRAS, ont porté sur $D$. transversa cv. "Waël ", ainsi que sur une plante de $D$. pilosiuscula et $11 \mathrm{cv}$. de $D$. trifida appartenant à la collection du Centre de Recherches Agronomiques des Antilles - Guyane (I.N.R.A. Guadeloupe). Les cultivars de $D$. trifida étudiés sont :

— «Torchon D » (forme archaïque de Guyane)
- « Tonate $1 \mathrm{E}$ », « Tonate 2B » et « Moengo 3 » (cultivars de Guyane)

- "Cousse Couche Violette" (cv. antillais traditionnel)

- «I.N.R.A. 16 ", "I.N.R.A. 23 " (hybrides du CRAAG en collection), "I.N.R.A. 25 ", « 2-104 » et «74-1-84 » (en cours d'étude agronomique) ainsi que différents hybrides, dont "I.N.R.A. $25 \times 5-20$ " aimablement fourni par M. MIGINIAC du CNRS (Gifsur-Yvette).

Ces dénombrements ont été réalisés sur des méristèmes radiculaires directement issus de la germination des tubercules (ou de bulbilles dans le cas de $D$. pilosiuscula) placés préalablement en atmosphère humide à $25^{\circ} \mathrm{C}$.

Les pointes de racines d'environ $1,5 \mathrm{~cm}$ sont placées, immédiatement et pendant 3 à $5 \mathrm{~h}$ selon leur grosseur, dans une suspension d' $\alpha$-monobromo- (ou chloro-) naphtalène préparée extemporanément. A l'issue de ce prétraitement, elles sont fixées dans l'alcool acétique $3: 1$ et conservées à environ $5^{\circ} \mathrm{C}$. La coloration est effectuée selon la technique de Feulgen après un lavage à l'eau distillée et une hydrolyse de $6 \mathrm{mn}$ dans l' $\mathrm{HCl} \mathrm{N}$ à $60^{\circ} \mathrm{C}$. Nous avons pu remarquer que l'étalement des préparations est ainsi amélioré par rapport à la méthode d'hydrolyse à température ambiante avec l' $\mathrm{HCl}$ 5N (ITIKAWA \& OGURA, 1954). Signalons cependant que cette dernière technique, qui peut se révéler utile, demande une durée d'hydrolyse de 20 à $25 \mathrm{mn}$ et qu'un rinçage à l' $\mathrm{HCl} \mathrm{N}$ est souhaitable avant l'immersion de $2 \mathrm{~h}$ dans le réactif de Schiff. Les pointes de racines sont alors soumises à un lavage soigneux avec de l'eau renouvelée plusieurs fois pendant 5 à $10 \mathrm{mn}$ traitées pendant $1 \mathrm{~h}$ par une solution de Drisélase (KYOWA HAKKo KogYo Co Ltd, Tokyo, Japon) à 0,5 p. 100 à la température ambiante. L'écrasement est effectué entre lame et lamelle dans une goutte de carmin acétique ferrique. Les préparations sont lutées avec une dissolution de caoutchouc et peuvent être conservées pendant quelques jours à environ $5{ }^{\circ} \mathrm{C}$ ou quelques semaines à $-18{ }^{\circ} \mathrm{C}$.

\section{RÉSULTATS}

Les difficultés rencontrées lors des dénombrements des 3 espèces étudiées vont croissant en passant de $D$. tranversa à $D$. trifida et à $D$. pilosiuscula.

Les dénombrements de $D$. transversa cv "Waël» ne présentent pas de difficultés particulières. Les chromosomes s'étalent relativement bien et s'individualisent sans trop de peine. On peut ainsi attribuer à ce cultivar un nombre $2 \mathrm{n}=80$ incontestable (fig. 2).

L'analyse des plaques métaphasiques de $D$. trifida (fig. 1a et 1b) est rendue beaucoup plus imprécise par suite d'un étalement chromosomique difficile ainsi que par l'existence d'associations entre chromosomes beaucoup plus fréquentes et d'interprétation plus délicate que dans le cas présent. C'est ainsi qu'on peut rencontrer des chaînes associant jusqu'à 6 chromosomes. Ces associations ont d'ailleurs été signalées très tôt dans le genre Dioscorea où elles se retrouvent dans bon nombre d'espèces (SUESSENGUTH, 1921, RAMACHANDRAN, 1968). Malgré ces difficultés, il 


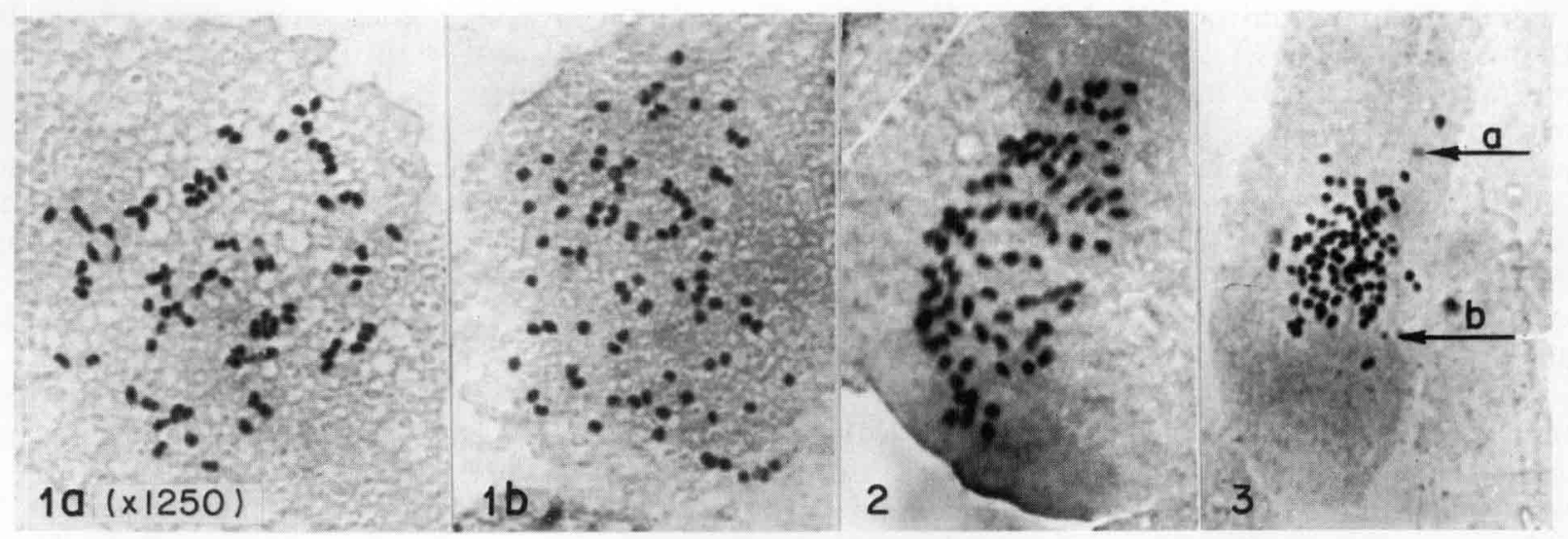

Figures 1 à 3

Plaques métaphasiques.

Metaphase plates.

$I a, I b:$ D. trifida $L . \quad(2 n=80)$

2: D. transversa Brown $(2 n=80)$

3: D. pilosiuscula Bert. $(2 n=$ environ 80$)$ avec 2 chromosomes douteux (explications dans le texte). $(2 n=c a 80)$ with two doubtful chromosomes (see text).

apparaît très nettement que les 11 cultivars de $D$. trifida analysés ici possèdent le même nombre de chromosomes $2 \mathrm{n}=80$.

Chez D. pilosiuscula, la complexité de l'analyse s'accroît encore considérablement en raison de la taille des chromosomes. Ceux-ci sont dans l'ensemble nettement plus petits que ceux des autres espèces de Dioscorea. Il suffit pour s'en convaincre de comparer la fig. 3 avec les fig. 1 et 2 du présent article et les photographies de RAMACHANDRAN (1968). Les plus petits éléments (fig. 3: flèche a) apparaissent en nombres variables dans les cellules (de 2 à 9 avec une fréquence maximum à 4). Nous les considérons $a$ priori comme des chromosomes B bien qu'il puisse parfois s'agir de satellites et, de ce fait, ils n'ont pas été introduits dans les dénombrements chromosomiques proprement dits. Ces petits éléments ne sont pas toujours faciles à distinguer des plus petits des autres chromosomes du stock dont certains sont parfois peu colorés (fig. 3 : flèche B) et ressemblent, dans certains cas, à des inclusions cellulaires banales.

Ces difficultés s'ajoutent à celles qui sont habituellement rencontrées dans le genre Dioscorea et qui expliquent la fréquence des dénombrementş approximatifs (cf. tabl. 1). Nos dénombrements nous permettent seulement d'estimer le nombre $2 \mathrm{n}$ de $D$. pilosiuscula à, très approximativement, 80 chromosomes.

Une autre remarque qui peut être faite à propos de D. pilosiuscula concerne l'absence de chromocentres dans les noyaux interphasiques. Cette structure s'oppose à celle rencontrée chez d'autres espèces du genre telles que $D$. trifida et $D$. transversa (voir aussi SUESSENGUTH, 1921 ; MEURMAN, 1925 ; SHARMA \& DE, 1956).

\section{DISCUSSION}

\section{A. Les nombres chromosomiques étudiés}

En ce qui concerne l'espèce alimentaire océanienne $D$. transversa, le nombre $2 \mathrm{n}=80$ n'appelle aucun commentaire particulier étant donné, d'une part, que ce dénombrement est original et, d'autre part, que cette espèce peut être considérée comme dérivant directement du groupe asiatique à $\mathrm{x}=10$ (BURKILL, 1960). «Waël » est donc un cultivar octoplö̈de $(2 \mathrm{n}=8 \mathrm{x})$ d'une espèce dont la variabilité chromosomique reste à établir.

Nos dénombrements de $D$. trifida sont en opposition évidente avec ceux présentés par HENRY (1967) et par MARTIN \& DEGRAS (1978) en ce qui concerne la variabilité des nombres chromosomiques de l'espèce, d'une part, et son nombre de base, d'autre part. En effet, la variabilité chromosomique des cultivars étudiés par HENRY apparaît comme considérable puisque 5 origines dénombrées se répartissent entre 3 niveaux de ploïdie $(2 n=54,72,81)$ qui ont un nombre de base $x=9$. En outre, MARTIN \& DEGRAS (1978) signalent l'existence de plantes à $2 \mathrm{n}=18,36$ et 54 chromosomes.

Or l'étude attentive de nos cultivars ( + divers hybrides), choisis (par L. DEGRAS) pour représenter autant que possible la variabilité de l'espèce, n'a pas permis de déceler l'existence de nombres de chromosomes différents de 80 , sauf quelques plaques présentant accidentellement des nombres de chromosomes inférieurs comme il est courant d'en obtenir dans le cours d'un travail de cet ordre. Il nous a donc été impossible de confirmer la série polyploïde de base 9 avancée par les auteurs précités dont nous n'avons malheureusement pu obtenir le matériel caractéristique.

D'autre part la fertilité de $D$. trifida est généralement excellente puisque cette espèce ...« may be the only cultivated yam species to retain sexuality fully » (MARTIN \& DEGRAS, 1978) : une telle fertilité ne s'accorde ni avec un degré de polyploïdie variable, ni surtout avec un état nanoploïde correspondant à 81 chromosomes. Par contre, un nombre de chromosomes $2 \mathrm{n}=8 \mathrm{x}=80$ ne s'opposerait qu'à l'hypothèse actuelle de l'absence, dans le continent américain, du nombre de base $\mathrm{x}=10$ (MARTIN \& ORTIZ, 1963).

En fait nous constatons :

1. La parfaite fertilité des descendants hybrides 
obtenus entre tous cultivars d'origines différentes (L. DEGRAS, comm. pers.) ce qui suppose

a) que tous ces cultivars ont un même nombre de chromosomes,

b) que la disjonction chromosomique en méiose est régulière, tant chez les parents que chez les descendants c'est-à-dire que tous les individus possèdent un nombre pair de génomes de base.

2. Que 2 espèces américaines à $\mathrm{x}=10$ ont déjà été signalées : il s'agit de $D$. dodecaneura Vell., à $2 \mathrm{n}=40$ (SMITH 1937 ; NAKAJIMA, 1942 ; MIEGE, 1952) et $D$. villosa L., à $2 \mathrm{n}=20$ (MEURMAN, 4925).

3. Que 24 espèces américaines seulement ont été dénombrées sur un nombre estimé à plus de la moitié des 600 espèces du genre (SMITH, 1937) (tabl. 1).

\section{B. Polyploïdie et nombre de base}

Compte tenu de la discussion qui précède, la récapitulation de l'ensemble des résultats de dénombrements chromosomiques dans le genre Dioscorea auxquels nous avons pu avoir accès est présentée dans le tableau 1 qui comprend 71 espèces différentes en tenant compte des synonymies exprimées par BURKILL (1960) et COURSEY (1967), sauf pour $D$. macroura Harms. et $D$. sansibarensis Pax (AYENSU, 1972). On notera la place particulière de $D$. bulbifera L. dont la présence en Asie et en Afrique s'accompagne de la coexistence des 2 nombre de base $\mathrm{x}=9$ et 10, caractère qui n'est apparemment partagé que par $D$. dumetorum et par $D$. trifida. Ces remarques ne sont bien évidemment encore que des tentatives d'éclaircissement de l'organisation du genre, les nombres chromosomiques connus ne constituant encore qu'une très petite fraction du total. On peut cependant admettre que les dénombrements de $D$. $t r i$ fida présentés ici confirment l'existence d'un nombre de base $x=10$ sur le continent américain qui s'ajoute au nombre $x=9$ précédemment admis. En effet, si l'on élimine l'espèce nord-américaine $D$. villosa L., susceptible de dériver directement des Dioscorea asiatiques selon BURKILL (1960), il reste encore 2 espèces d'Amérique du Sud et d'Amérique Centrale à $\mathrm{x}=10: D$. dodecaneura Vell. et $D$. trifida. Il n'est peut-être pas sans intérêt de remarquer que ces 2 espèces sont les seules espèces d'ignames alimentaires américaines autochtones signalées par COURSEY (1967), à nombres chromosomiques connus ; $D$. trifoliata Grisebach, n'a pas encore à notre connaissance, été dénombrée.

Le tableau 2 met en évidence de façon statistique la répartition des niveaux de ploïdie dans le genre Dioscorea. Ce tableau découle du tableau 1 mais ne prend pas en compte les nombres indiqués par les auteurs eux-mêmes comme douteux (annotations ca du tableau 1). On voit ainsi apparaître les caractéristiques majeures de l'évolution chromosomique du genre Dioscorea :

1. La polyploïdie y participe d'une façon prédominante soit de façon autonome (formes $4 x, 8 x$ et peutêtre $16 \mathrm{x}$ ), soit en association avec l'hybridation puisqu'on observe tous les intermédiaires entre $2 \mathrm{x}$ et $10 \mathrm{x}$, ce qui est particulièrement frappant pour D. alata (tabl. 1).

2. L'importance du phénomène apparaît nettement si l'on considère que les formes $2 \mathrm{x}$ ne représentent, d'après les données actuelles, que 13 p. 100 du total.

3. Les niveaux de ploïdie les plus fréquents sont $4 \mathrm{x}$ (43 p. 100), 6x (13 p. 100) et $8 \times(9$ p. 100). Cette distribution, qui n'est pas surprenante chez les végétaux supérieurs, pourrait correspondre à l'existence de valeurs adaptatives optimales susceptibles de varier avec le génotype mais qui n'ont pu encore être expérimentalement définies (SHARMA \& DE, 1956).

4. Les niveaux de ploïdie les plus élevés que l'on rencontre dans le Nouveau comme dans l'Ancien Monde ne sont pas l'apanage des espèces alimentaires (caractères gras et italiques du tableau 1). C'est ainsi qu'on peut relever dans le tableau 1 l'existence de 6 espèces considérées comme non alimentaires à $2 \mathrm{n} \geq 10 \mathrm{x}$ et, inversement, on voit que $D$. dumetorum et $D$. alata ne dépassent pas, semble-t-il, $6 \mathrm{x}$ et $8 \mathrm{x}$ respectivement.

5. Si l'on prend $\mathrm{x}=10$ comme nombre de base caractéristique, on s'aperçoit que ce nombre est valable en Asie pour 100 p. 100 des espèces dénombrées mais ne correspond plus qu'à 60 p. 100 des espèces africaines et à 14 p. 100 seulement des espèces américaines, le complément étant apporté par les espèces à $\mathrm{x}=9$.

\section{CONCLUSIONS}

Dans le genre Dioscorea on peut donc conclure à une association évolutive apparente de la polyploïdie avec une tendance à la modification du nombre chromosomique de base. Il apparaît en outre que si un degré élevé de polyploïdie est compatible avec la survie, au moins dans certaines situations, ces formes à $2 n \geq 10 x$ peuvent être considérées comme des culsde-sacs évolutifs, au contraire des formes à polyplö̈die réduite ou intermédiaire. Ce qui, nous semble-t-il, ne fait qu'illustrer, mais d'une façon particulièrement claire, l'interaction d'un comportement individuel perenne avec, d'une part, les possibilités d'évolution rapides mais limitées offertes par la polyploïdie et, d'autre part, les exigences génétiques d'une évolution plus profonde mais nécessairement plus lente et probablement encore ralentie par le processus même de la polyploïdie (cf. ESSAD, 1957; DARLINGTON, 1963). C'est probablement ce mécanisme d'évolution tamponnée dans un milieu fortement conservateur qui nous vaut de trouver ici une image particulièrement fidèle des processus évolutifs fondamentaux qui régissent le règne végétal. $\mathrm{Ce}$ qui ne fait qu'ajouter à l'intérêt économique (alimentaire et pharmacologique) déjà considérable du genre Dioscorea.

Il paraît, d'autre part, extrêmement souhaitable :

1. Que des caryologistes confirmés élargissent l'étude des cultivars de D. trifida pour établir définitivement le statut chromosomique de cette espèce importante. 
TABLEAU 1

Les nombres chomosomiques dans le genre Dioscorea.

En italiques : espèces alimentaires. En caractères gras : espèces alimentaires d'importance majeure. SE = présent travail.

Chromosome numbers in the genus Dioscorea.

Italics : Food species. Bold face : Major food species. SE = present study.

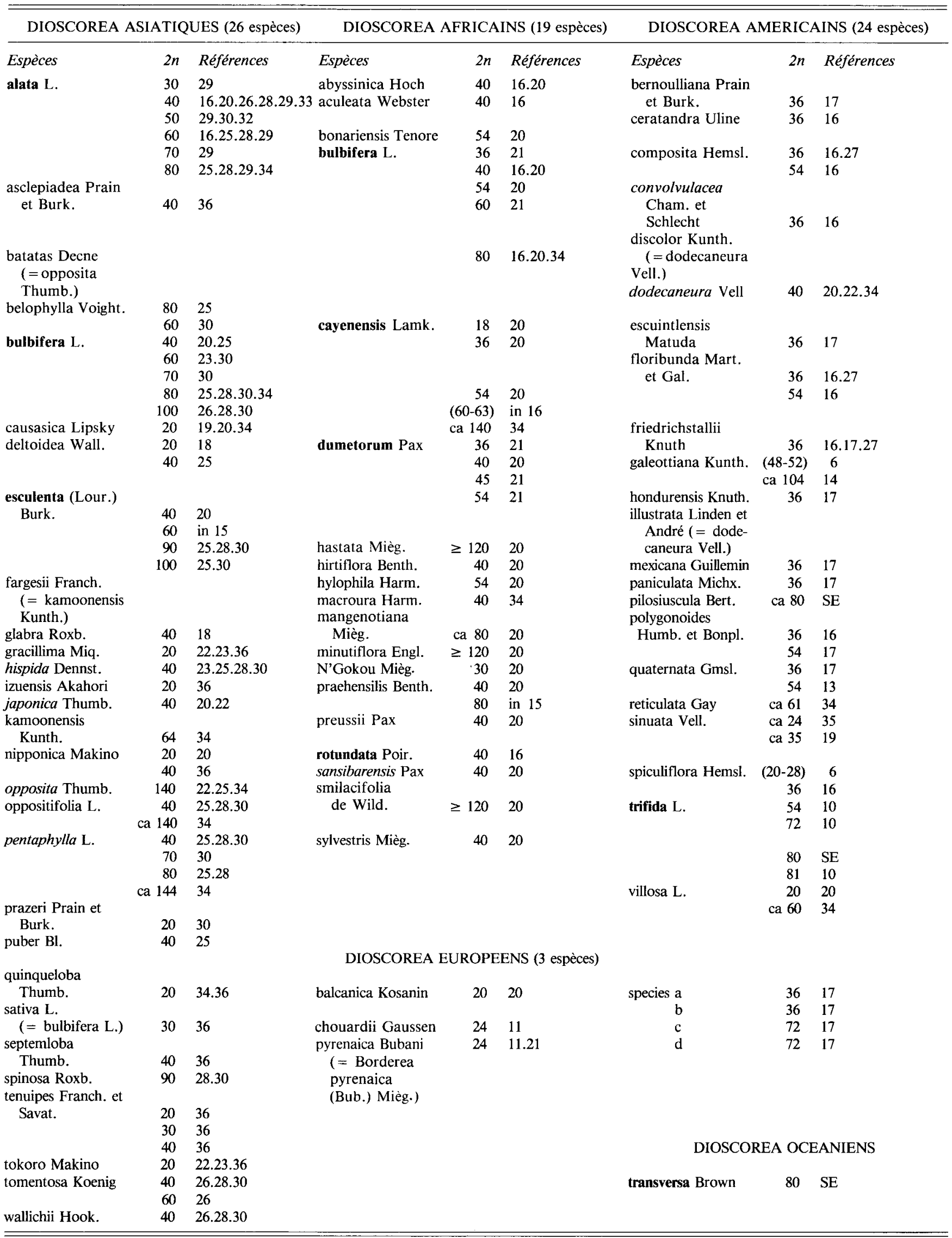


Répartition des niveaux de ploidie dans le genre Dioscorea. Nombre de cas observés et, en caractères gras, les pourcentages correspondants. Dans la colonne «Total », les chiffres entre parenthèses représentent les cas où les nombres de chromosomes proposés $(<10 \mathrm{x})$ ne permettent pas l'attribution d'un nombre de base $x$ (comme pour les nombres $>10 \mathrm{x}$ ).

Ploidy level in the genus Dioscorea. Numbers of observed cases and, in bold type, the corresponding percentages. In the column "Total ", the brackets enclose cases where proposed chromosome numbers $(<10 \mathrm{x})$ do not allow the attribution of a base number $x$ (as for the numbers $\geq 10 \mathrm{x}$ )

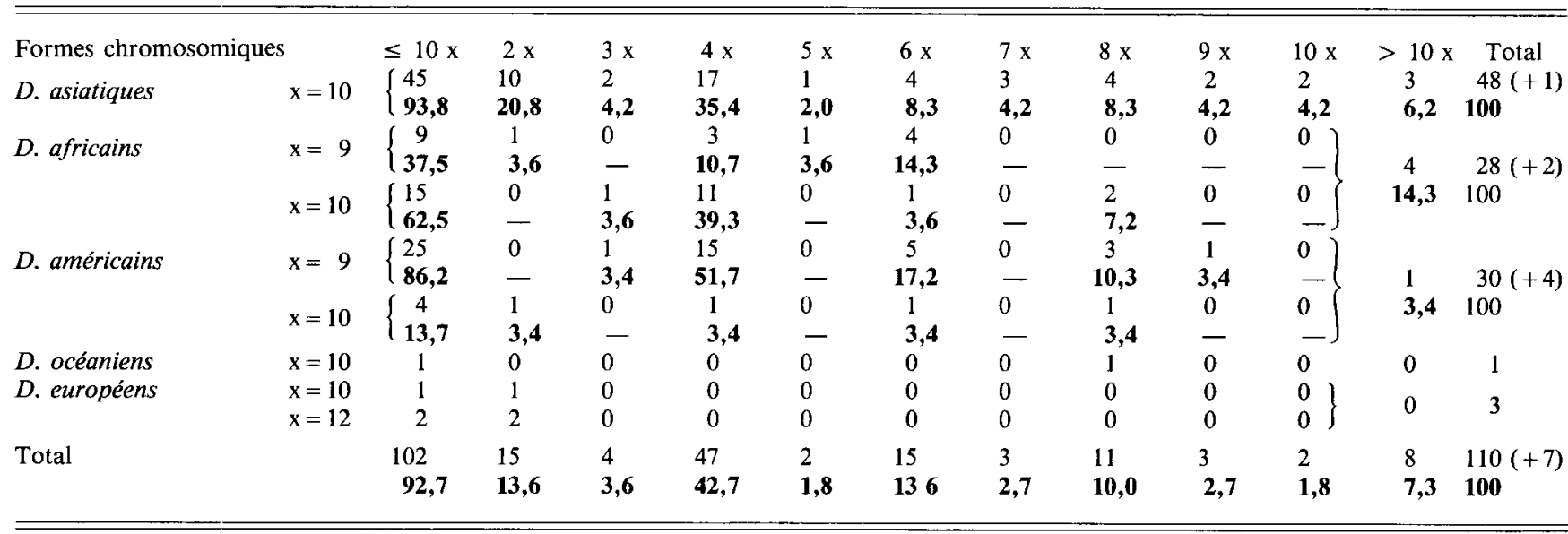

2. De mettre en doute l'hypothèse généralement admise selon laquelle les Dioscorées américaines ne présenteraient qu'un seul nombre chromosomique de base.

Reçu le 25 novembre 1983. Accepté le 9 mars 1984

\section{REMERCIEMENTS}

L. Degras a été à l'origine de cette étude et y a contribué de façon essentielle par la fourniture du matériel végétal et par sa critique du texte présenté. Qu'il veuille bien trouver ici l'expression de mon amitié.

\section{RÉFÉRENCES BIBLIOGRAPHIQUES}

1. Ayensu E. S., 1972. Anatomy of Monocotyledons. Dioscoreales. Clarendon Press, Oxford, $182 p$

2. Ayensu E. S., Coursey D. G., 1972. Guinea Yams. Econ. Bot., 26, 301-318.

3. Burkill I. H., 1960. The organography and the evolution of Dioscoreaceae, the family of the yams. J. Linn. Soc. Bot., 56, 319412 .

4. Coursey D. G., 1967. Yams. Longman, London, 230 p.

5. Coursey D. G., 1976. Yams. Dioscorea spp. (Dioscoreaceae), 70-74. In Simmonds N. W. : «Evolution of crop plants », Longman, London and New York, 339 p.

6. Cox D. K., Corzo A. H., Matuda E., Duran J. G. G., 1958 Estudio de las Dioscoreas mexicanas. I. Dioscorea spiculiflora Hemsl. Bol. Soc. Bot. Mexico, 22, 1-16.

7. Darlington C. D., 1963. Chromosome botany and the origin of cultivated plants. G. ALLEN and UNwIN Ltd., London, $231 \mathrm{p}$.

8. Degras L., Arnolin R., Poitout A., Suard C., 1977. Quelques aspects de la biologie des Ignames (Dioscorea spp.). I. Les ignames et leur culture. Ann. Amélior. Plantes, 27, 1-23.

9. Essad S., 1957. La polyploïdie et ses aspects évolutifs en relation avec l'amélioration des plantes. Ann. Amélior. Plantes, 7, 199-226.

10. Henry V. C. R., 1967. Studies on botanical and agronomic characteristics in cush-cush (Dioscorea trifida L.). Thesis Dr. Phil., Dept Agronomy. McGill Univ. Montréal, July 1967.

11. Heslot H., 1953. Le nombre chromosomique des Dioscoreacées pyrénéennes et leur rattachement au genre Borderea Miég. $C$. $R$ Acad. Sci., Paris, 237, 433-434

12. Itikawa O., Ogura Y., 1954. The Feulgen reaction after hydrolysis at room temperature. Stain Technol., 29, 13-15.
13. Jensen H. W., 1937. Meiosis in several species of dioecious Monocotyledonae. I. The possibility of sex chromosomes. Cytologia Fujii Jub. Vol., 96-103.

14. Leal F. M., 1959. Anatomia floral y embriogenia en Dioscored galeottiana Kunth. Acta Cient. Potosina, 3, 9-52.

15. Martin F. W., Degras L., 1978. Tropical yams and their potential. Part 5. Dioscorea trifida. USDA Agric. Handb., $\mathrm{N}^{\circ} 522$, $26 \mathrm{p}$.

16. Martin F. W., Ortiz S., 1963. Chromosome number and behaviour in some species of Dioscorea. Cytologia, 28, 96-101.

17. Martin F. W., Ortiz S., 1966. New chromosome numbers in some Dioscorea species. Cytologia, 31, 105-107.

18. Mehra P. N., Sachdeva S. K., 1976. Cytological observations on some W himalayan monocots. IV. Several families. Cytologia, 41, 31-53.

19. Meurman 0., 1925. The chromosome behaviour of some dioecious plants and their relatives with special reference to sex chromosomes. Soc. Sci. Fenn. Comm. Biol., 2, 1-105.

20. Miege J., 1952. Contribution à l'étude systématique des Dioscorea d'Afrique occidentale. Thèse Paris, $266 \mathrm{p}$.

21. Miege J., 1954. Nombre chromosomique et répartition géographique de quelques plantes tropicales et équatoriales. Rev. Cytol. Biol. Vég., Paris, 15, 312-348.

22. Nakajima G., 1934. Chromosome numbers in some Angiosperms. Jap. J. Genet., 10, 1-5.

23. Nakajima G., 1937. Cytological studies in some dioecious plants. Cytologia, Fujii Jub. Vol., 282-292.

24. Nakajima G., 1942. Cytological studies in some flowering dioecious plants with special reference to the sex chromosomes. Cytologia, 12, 262-270. 
25. Raghavan S. R., 1958. A chromosome survey of Indian Dioscorea. Proc. Indian Acad. Sci., ser. B, 48, 59-63.

26. Raghavan S. R., 1959. A note on some South Indian species of the genus Dioscorea. Current Sci., 28, 337-338.

27. Rama Rao V., Murty U. R., 1975. Meiotic studies in species and hybrids in medicinal yams. Current Contents, 44, 24-25.

28. Ramachandran K., 1962. Studies on the cytology and sex determination of the Dioscoreaceae. J. Indian Bot. Sci., 41, 93-98. 29. Ramachandran K., 1968. Cytological studies in Dioscoreaceae. Cytologia, 33, 401-410.

30. Sharma A. K., 1970. Annual report 1967-1968 (Cytogenetics Lab.). Res. Bull. Univ. Calcutta, 2, 1-50.

31. Sharma A. K., De D. N., 1956. Polyploidy in Dioscorea. Genetica, 28, 112-120.
32. Sharma A. K., Sharma A., 1957. Investigations leading to a new theory of differentiation in plant cells. Genet. Iber., 9, 143162.

33. Simmonds N. W., 1954. Chromosome behaviour in some tro pical plants. Heredity, 8, 139-146.

34. Smith B. W., 1937. Notes on the cytology and distribution of the Dioscoreaceae. Bull. Torrey Bot. Club, 64, 189-197.

35. Suessenguth K., 1921. Bemerkungen zur meiotischen und somatischen Kernteilungen bei Monocotylen. Flora N. F., 14, 313328.

36. Takeuchi Y., Iwao T., Akahori A., 1970. Chromosome numbers of some Japanese Dioscorea species. Acta Phytotax. Geobot., 24, 168-174. 